\title{
Application of X-Ray Fluorescence Analysis in Investigations of Historical Monuments
}

\begin{abstract}
T. Čechák, L. Musílek, T. Trojek, I. Kopecká
Nuclear techniques and other techniques using ionising radiation represent a valuable tool in non-destructive diagnostics applied to archaeological finds and objects of arts, namely for determining the composition of materials used in the production of artefacts. X-ray fluorescence analysis, both in its energy form and in its wave dispersive form, is one of the most widespread methods using ionising radiation to study the elemental composition of materials. It is frequently used for studies of various cultural and historic relicts and objects of art. This work summarizes the authors' experience with X-ray fluorescence analysis in investigating historical frescos namely by means of portable provide spectroscopic devices. The results of these measurements information on the composition of the pigments, enable the comparison of processes used in the fabrication of pigments by individual artists, and in many cases offer information on how to repair the damaged parts.
\end{abstract}

Keywords: X-ray Fluorescence Analysis, Si(Li) detectors, portable spectrometers, energy dispersive analysis, fresco paintings, ${ }^{55} \mathrm{Fe},{ }^{238} \mathrm{Pu}$.

\section{Introduction}

The preservation of artefacts, monuments, and archaeological sites and finds frequently requires scientific analysis of cultural materials or testing of specific properties of chemical treatments in order to document their historic evidence, clarify deterioration processes, and specify conservation treatments. Analyses of the variety of materials constituting our cultural heritage require the expertise of many various scientific methods. X-ray Fluorescence Analysis, as one of them, is a non-destructive analytical technique used to determine the elemental composition of a sample.

\section{Basic pronciple of X-ray fluorescence}

When a primary $\gamma$ or X-ray excitation beam from a radioactive source or from an X-ray tube strikes a sample, the photons can either be absorbed by the atom or scattered through the material. The process in which a photon is absorbed by the atom by transferring all of its energy to an innermost electron is called the photoeffect. During this process, if the primary photon has sufficient energy, electrons are ejected from the inner shells, creating vacancies. These vacancies present an unstable condition for the atom. When this atom returns to its stable state, an electron from the outer shell is transferred to the inner shell and in this process a characteristic X-ray is emitted, whose energy is equal to the difference between the two binding energies of the electrons in the corresponding shells. Each element has a unique set of energy levels each element produces X-rays with a unique set of energies allowing one to measure the elemental composition of the sample. Between the proton number $\mathrm{Z}$ of the atom emitting characteristic radiation and the energy, or the wavelength of the radiation, the following relationship applies

$$
E=k(\mathrm{Z}-b)^{2},
$$

where $E$ is the energy of the photons corresponding to the transfer between two specific shells, and $k$ and $b$ are constants. The process of emission of the characteristic X-rays is called
X-ray Fluorescence (XRF), and the analytical method using $\mathrm{XRF}$ is called X-ray Fluorescence Analysis (XRFA) [1].

\section{Analysis of fresco pigments}

The X-ray fluorescence equipment was built and is now operated in the Laboratory of Quantitative Methods in the Research of Ancient Monuments at FNSPE. For the in-situ measurements we built an XRF analyser with changeable radionuclide sources in the measuring head and with an $\mathrm{Si}(\mathrm{Li})$ detector (see Fig. 1). The radioisotope sources ${ }^{55} \mathrm{Fe}$, ${ }^{238} \mathrm{Pu}$, and ${ }^{241} \mathrm{Am}$, are used. ${ }^{55} \mathrm{Fe}$ enables the excitation of elements with low $\mathrm{Z}$ up to $23,{ }^{238} \mathrm{Pu}$ is used for the excitation of

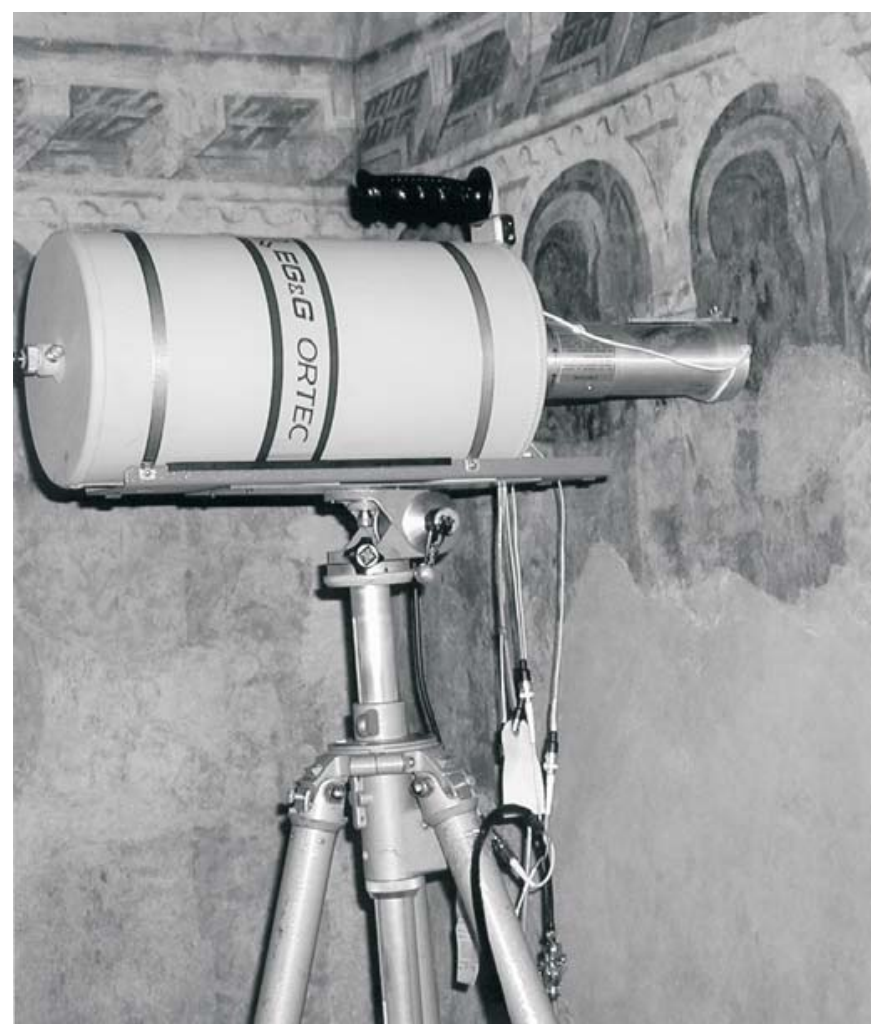

Fig. 1: $\mathrm{Si}(\mathrm{Li})$ detection equipment during the measurement of a fresco 
elements with $\mathrm{Z}$ from 20 up to 39 , and ${ }^{241} \mathrm{Am}$ is used for the excitation of the $\mathrm{K}$ shell electrons of elements with higher $\mathrm{Z}$ up to 68 . The $\mathrm{Si}(\mathrm{Li})$ detector is cooled by liquid nitrogen from a 51 Dewar vessel. For special purposes, a 21 Dewar vessel is also available. These small Dewar vessels and the portable multichannel analyser enable in situ measurements. The collimator system of the exited radiation makes it possible to select the irradiated area. Our spectrometer can be used for area mapping or line scanning.

Pigment compositions on fresco paintings vary with the locality and historic period. White pigments, for example, can be produced with $\mathrm{Pb}, \mathrm{Zn}$ and Ti oxides, but $\mathrm{ZnO}$ was not produced before 1870, and Titanium White was not used before 1920 [2]. Therefore, if either $\mathrm{Zn}$ or Ti is found in the white areas of fresco paintings supposed to be from the Renaissance
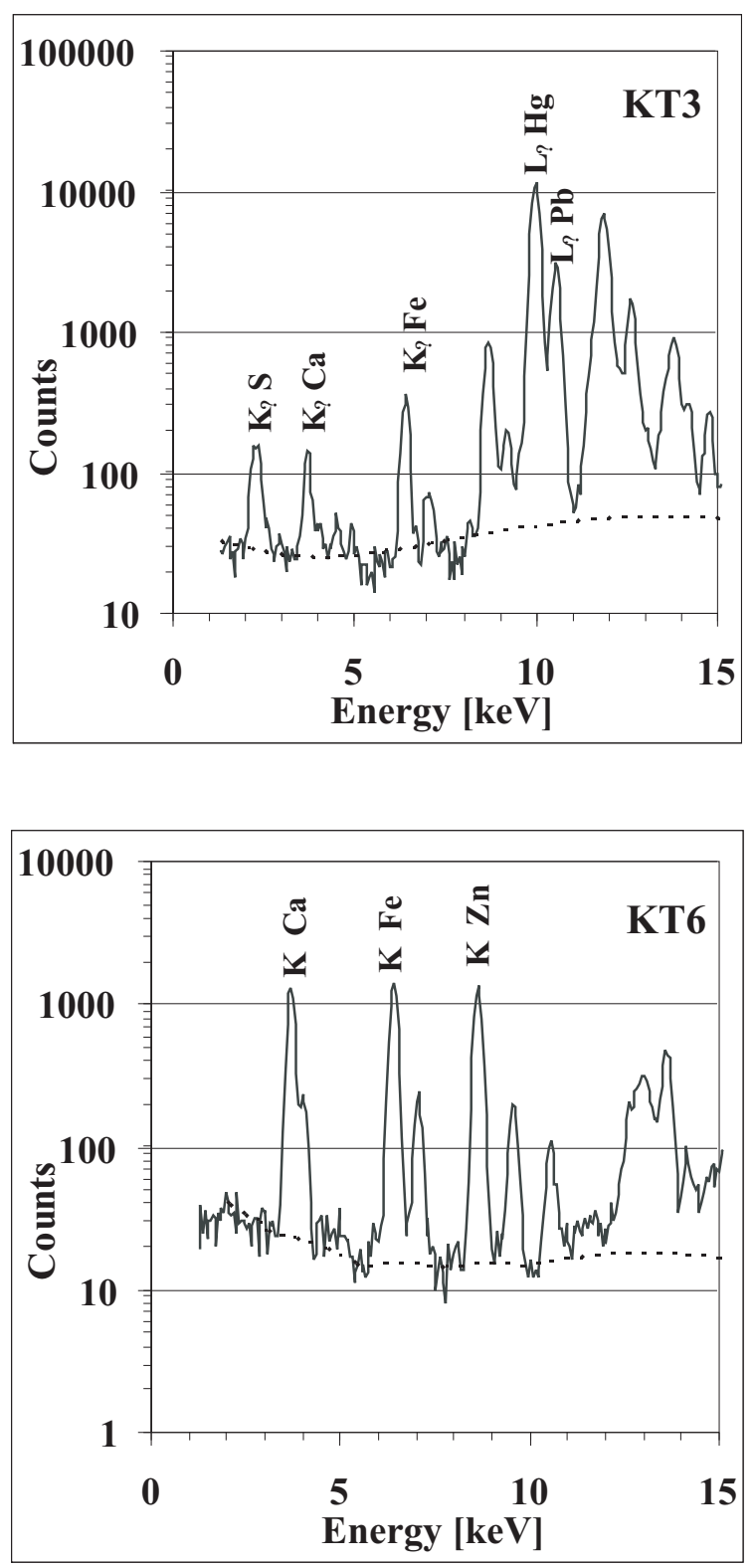

Fig. 2: XRF spectrum of the red pigments in the fresco in Karlštejn castle. In red pigment used in the 14th century was a mixture of Vermilion, Saturn Red and Red Ochre (KT3). Red Ochre with Chinese White was used as a red pigment in the $19^{\text {th }}$ century (KT6). period, those paintings were restored or they are a forgery. A more subtle study of the fresco paintings of several works of a particular artist to determine the pigments characteristically used by him or by his disciples would reveal frescos that were particularly typical for the artist and possibly, the times. Differences between countries or areas and time periods would most certainly be revealed, and differences between works of fresco painters in the same place at the same time period might be shown up, too.

The overall results of XRFA on frescos are multielement spectra indicating the main elements of the pigments that are present. Usually, this step makes it possible to identify the inorganic pigments under consideration. In many cases useful details about minor and trace elements can be obtained [3].

Several measurements have been carried out directly in the field in order to verify the method and receive information about Gothic fresco paintings. The valuable fresco paintings from Žirovnice Castle were investigated in this way. Surprisingly many pigments were used on these frescos, which was not typical of the Bohemian region at the end of $15^{\text {th }}$ century. For all basic paints, a few pigments and combinations of pigments were used. Local common pigments e.g., Green Earth and Yellow Ochre were used together with expensive imported pigments such as Vermilion, Saturn Red and Azurite and with pigments which were used very seldom on fresco paintings, e.g., Antimonate Yellow, and Manganese Brown.

Our investigation of the fresco paintings at Karlštejn Castle aims to date the particular parts of the frescos restored in the $19^{\text {th }}$ century, during the reconstruction of the castle. Analysis of XRF spectra of the pigments can give information about the type of pigments. Fourteenth century painters used other types of pigments than in the $19^{\text {th }}$ century, and the use of XRFA enables us to differentiate mediaeval and new parts of the fresco.

The red and black pigment was used as a marker of the mediaeval part of the fresco, see Fig. 2. The red pigment used in the $14^{\text {th }}$ century was a mixture of Vermilion, Saturn Red, and Red Ochre. Red Ochre with Chinese White was used as a red pigment in the $19^{\text {th }}$ century. A mixture of Mars Black with Saturn Red and Verdigris was used for tinting the black pigment in the $14^{\text {th }}$ century. This pigment differs from the black pigment used in the $19^{\text {th }}$ century (Mars Black with traces of $\mathrm{Zn})[4,5]$.

\section{Analysis of layered structures}

XRF facilities can also measure the thickness and perform a composition analysis of coating layer. The covering layer on certain areas can in many cases have a more or less constant thickness. The aim of this type of layer analysis is to separate qualitatively the presence of pigments from the covering layer and to identify the pigments and their position in the substrate layer by means of a non-destructive technique [6].

The basic principle of the measurements of thickness $h$ of a coating deposited on a substrate material is given by the following equation. For a given excitation spectrum, the intensity of the fluorescence radiation emitted from the substrate layer and measured by a detector depends on the angle of excitation $\psi_{i}$ and the angle of detection $\psi_{f}$, with $h$ as a parameter. In fact, the total fluorescence emitted by the sub- 
strate depends on the thickness of the substrate layer it is now assumed that the thickness is saturated, i.e. it is so big that the signal does not depend on it. The intensity of the measured fluorescence emitted from the substrate can easily be calculated for a simple model defined by the following condition: a single-element coating deposit and a single element substrate, monochromatic excitation at energy $E_{0}$, a flat and smooth surface area, a substrate of infinite thickness and only primary fluorescence are considered.

Under this assumption, the fluorescence intensity $I_{S F}$ measured by the detector is given by

$$
\begin{aligned}
I_{S F}= & I_{0} \varepsilon \tau\left(E_{0}\right) \frac{\Delta \Omega}{4 \pi} \eta\left(E_{S}\right) \\
& \left.\cdot\left\{1-\exp \left\{-\frac{\mu_{C}\left(E_{0}\right)}{\sin \psi_{i}}+\frac{\mu_{C}\left(E_{S}\right)}{\sin \psi_{f}}\right] \rho_{C} h\right\}\right\} \\
& \times \frac{1}{\rho_{S} \sin \psi_{i}} \frac{1}{\frac{\mu_{S}\left(E_{0}\right)}{\sin \psi_{i}}+\frac{\mu_{S}\left(E_{S}\right)}{\sin \psi_{f}}}
\end{aligned}
$$

where $I_{0}$ is the intensity of the incident radiation, and $\varepsilon$ is the excitation factor for the respective X-ray line of the substrate.

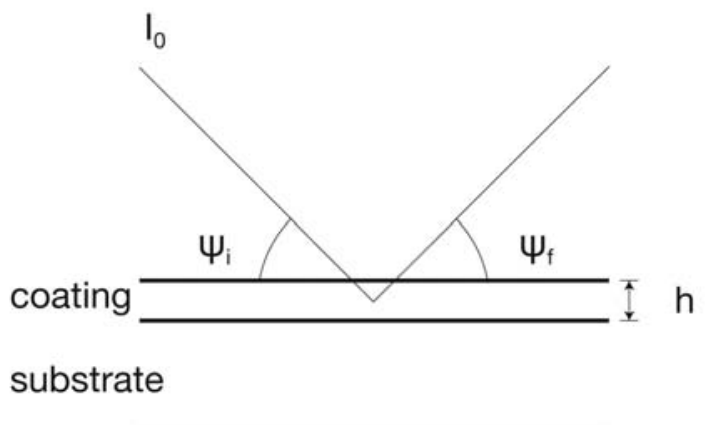

Fig. 3: Geometry of the experiment

When considering a $K$ line, $\varepsilon$ is the product of the absorption jump factor for the creation of a $K$ vacancy, the fluorescence yield for the decay of the $K$ vacancy, and the relative emission rate for the specific $K$ line with respect to other $K$ lines. $\tau\left(E_{0}\right)$ is the photoelectric absorption coefficient of the substrate at energy $E_{0} ; \Delta \Omega / 4 \pi$ is the fraction of the emitted photons collected within the detector solid angle, $\eta\left(E_{S}\right)$ is the detection efficiency at the fluorescence energy of the substrate $E_{S} ; \rho_{S}$ is the density of the substrate; $\rho_{C}$ is the density of the coating; and $\mu_{S}\left(E_{0}\right)$ and $\mu_{S}\left(E_{S}\right)$ are mass absorption coefficients of the substrate at energies $E_{0}$ and $E_{S}$, respectively and $\mu_{C}\left(E_{0}\right)$ and $\mu_{C}\left(E_{S}\right)$ are the mass absorption coefficients of the coating at energies $E_{0}$ and $E_{S}$ respectively [6].

In principle, knowing the coating density, it is possible for this very simple model to determine analytically also the coating thickness h, by using the measured intensities. From the model, we can to define the energy of the primary radiation what is necessary for irradiation of the atoms in the substrate layer and $\mathrm{Z}$ of the atoms whose characteristic radiation can be detected.

Transmission of characteristic $\left(K_{\alpha}\right.$ and $K_{\beta}, L_{\alpha}$ and $\left.\mathrm{L}_{\beta}\right)$ radiation through a layer of painting material will result in modification of the intensity ratio $I_{\alpha} / I_{\beta}$. This effect becomes obvi- ous if the attenuation is sufficiently different for the $\alpha$ and $\beta$ components of the excited radiation. Maximum $I_{\alpha} / I_{\beta}$ modification is anticipated for selective absorption, i.e., if the element of strongest absorption in the pigment ( $\mathrm{Hg}$ in Cinnabar and $\mathrm{Pb}$ in Lead White) has an absorption edge just between the $K_{\alpha}$ and $K_{\beta}$ or $L_{\alpha}$ and $\mathrm{L}_{\beta}$ energy of the penetrating radiation [7]. In order to search for pigment combinations, i.e. top layer - subjacent layer, where layer position studies via the $K_{\alpha} / K_{\beta}$ or $L_{\alpha} / \mathrm{L}_{\beta}$ intensity ratios are practicable, estimations are very useful on the basis of single elements. For this, modification of the intensity ratio $I_{\alpha} / I_{\beta}$ is expressed by a gain factor for the characteristic element of the pigment:

$$
g=\frac{I_{\alpha}(e, a) / I_{\beta}(e, a)}{I_{\alpha}(e) / I_{\beta}(e)},
$$

where $(e, a)$ represents the emitter - absorber layer system and $(e)$ denotes only the emitter without the attenuating top layer. It is well known that this gain factor

$$
g(S)=\exp (S d)
$$

is a function of the absorption selectivity:

$$
S=\left[\left(\frac{\mu}{\rho}\right)_{\alpha}-\left(\frac{\mu}{\rho}\right)_{\beta}\right] \rho
$$

where $\left(\frac{\mu}{\rho}\right)_{\alpha}$ and $\left(\frac{\mu}{\rho}\right)_{\beta}$ denote the mass attenuation coefficients for the considered system, and $\rho$ and $d$ represent the top material density and thickness, respectively [8].

For a realistic situation, the two models mentioned above are too simple. The Monte Carlo method must be used for calculating the intensities of the $K$ and $L$ lines of characteristic radiation from the subjacent layer. The MCNP-IVC code is very useful for this calculation [9], and this code is available and used in our laboratory.

In the Franciscan Monastery in Kadaň, an extremely precious Gothic fresco was found. Unfortunately, this fresco was superimposed by a new layer of fresco in the Renaissance period. The Renaissance fresco is also valuable and removing it will be very hazardous and expensive. XRFA can help here to investigate the subjacent layer of the Gothic fresco.

\section{Conclusions}

For elemental analysis of the pigments used in fresco-paintings, XRFA is the preferred technique for routine work. This is because of the mobility of the equipment, which ensures that the object can be kept in its stationary position during the measurement. Compared to other techniques, XRFA has the advantage of being non-destructive, multi-elemental, fast, and cost-effective. Thanks to advances in miniaturization of electronics, detectors, cooling equipment and X-ray tubes, it is possible to build and use portable equipment for XRFA.

XRFA along with thermoluminiscence dating will remain an important technique used in the Laboratory of Quantitative Methods in the Research of Ancient Monuments at FNSPE. In the future research work, the use of small X-ray tubes is also planned for studies of fresco paintings. The use of X-ray tube beams can increase the area resolution of the method. Better collimation of the beam enables the applica- 
tion of more precise software for quantitative analysis. Thanks to fruitful collaboration with the National Institute for Monument care, it is possible to compare the XRFA results with the results obtained by other methods, e.g. electron microscopy and, last but not least, collaboration with the PIXE laboratory of FNSPE also gives some interesting results.

Pigment analysis is also an extremely important aid in restoration, since it can help to distinguish the original sections of painting from the restored or later added sections. Thus the pigment characterisation may be very important in making a decision whether to remove spurious layers or for choosing the most closely matching process for restoration. The main reason for analysing pigments in frescos or in manuscripts is for the purposes of conservation. Depending on their nature, pigments may be sensitive to light, humidity, gaseous atmospheric pollutants, or heat, which requires specific storage or display conditions. Additionally, we may want to identify the pigments before applying some chemical or other treatment aimed at reversing or at least putting a stop to the deterioration process. Characterisation of the pigments may also help in assigning a probable date to the painting, in reconstructing its restoration and conservation history, and in detecting forgeries.

\section{References}

[1] Van Grieken, R. E., Markowicz, A. A. (eds.): Handbook of $X$-Ray Spectrometry. $2^{\text {th }}$ Edition, Marcel Dekker, New York 2002.

[2] Šimunková, E., Bayerová, T.: Pigmenty. Společnost pro technologie ochrany památek, STOP Praha 1999.

[3] Frankel, R.: "Detection of Art Forgeries by X-Ray-Fluorescence Spectroscopy." In: Isotopes and Radiation Technology, Vol. 8 (1970), No. 1.

[4] Čechák, T., Gerndt, J., Musílek, L., Kopecká, I.: “Application of X-Ray Fluorescence for Analysis of Fresco Paintings.” In: European Conference on Energy Dispersive X-Ray Spectrometry. Krakow : FAST PLK, 2000, Vol. 1, p. 167-169.

[5] Musílek, L., Čechák, T., Kopecká, I.: "X-Ray Fluorescence in Research on the Cultural Heritage." In: $1^{\text {st }}$
iTRS International Symposium on Radiation Safety and Detection Technology. Seoul: Korean Nuclear Society, 2001, Vol. 1, p. 228-234.

[6] Fiorini, C. et al.: "Determination of the Thickness of Coating by Means of a New XRF Spectrometer." X-ray Spectrometry, Vol. 31 (2002), p. 92-99.

[7] Mantler, M., Schreiner, M.: "X-Ray Fluorescence Spectrometry in Art and Archeology." X-ray Spectrometry, Vol. 29 (2000), p. 3-17.

[8] Neelmeijer, C. et al.: "Paintings - a Challenge for XRF and PIXE Analysis.” X-Ray Spectrometry, Vol. 29 (2000), p. 101-110.

[9] MCNP IVC Manual, http://www.jlab.org/ semenov/ rlinks/soft.html

Prof. Ing. Tomáš Čechák, CSc.

phone: +420222314132

fax: +420224811074

e-mail: cechak@fjfi.cvut.cz

Prof. Ing. Ladislav Musílek, CSc.

phone: +420224358247

fax: +420224811074

e-mail:musilek@fjfi.cvut.cz

Ing. Tomáš Trojek

phone: +420224358242

fax: +420224811074

e-mail: tomas.trojek@fjfi.cvut.cz

Czech Technical University in Prague

Faculty of Nuclear Sciences and Physical Engineering,

Břehová 7

11519 Prague 1, Czech Republic

Ing. Ivana Kopecká, CSc.

phone: +420224 213813

fax: +420224232025

e-mail:kopecka@praha.npu.cz

Národní památkový ústav

Valdštejnské nám. 3

11801 Prague 1, Czech Republic 